\title{
Case Report: Post-traumatic Anton's Syndrome and Its Rehabilitation Challenges: A Case Report
}

\author{
Kavitha Andiappan ${ }^{1^{*}}$ (D) \\ 1. Department of Rehabilitation Medicine, Hospital Sungai Buloh, Selangor, Malaysia.
}

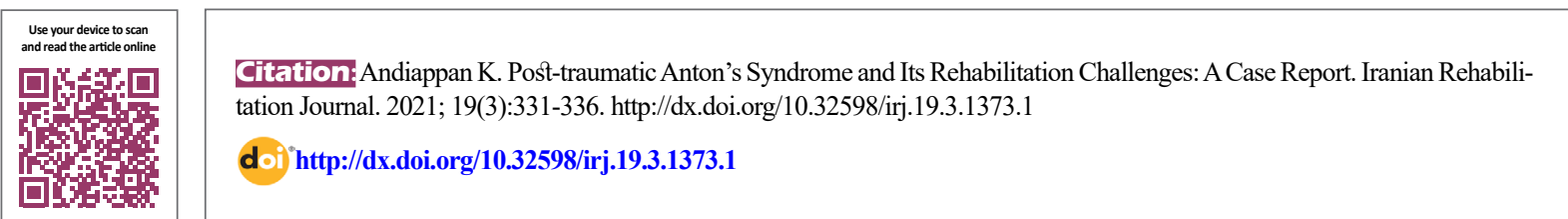

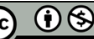

Article info:

Received: 15 Mar 2021

Accepted: 06 Jul 2021

Available Online: 01 Sep 2021

\section{Keywords:}

Rehabilitation, Anton's syndrome, Traumatic brain injury

\section{A B S T R ACT}

Objectives: Anton's syndrome is a rare clinical phenomenon characterized by acquired cortical blindness without the self-awareness of blindness. Patients with this syndrome tend to deny their visual deficits. The literature on the rehabilitation of individuals with Anton's syndrome is sparse.

Methods: We describe a 26-year-old patient who developed Anton's syndrome in the background of anterior visual tract damage and multiple intracranial bleedings after traumatic brain injury. He presented with visual agnosia and confabulations that were only detected 2 months after trauma. He received 4 months of multidisciplinary rehabilitation care as an outpatient. His comprehensive program consisted of (a) compensatory strategies, (b) restorative strategies, and (c) counseling sessions. Although he showed significant improvement in terms of functional gain with rehabilitation, he still had visual deficits with occasional visual anosognosia that required 24-h supervision.

Conclusion: Anton's syndrome in traumatic brain injury should not be overlooked. A prompt diagnosis is vital to initiate appropriate rehabilitation measures. 


\section{Highlights}

- Head trauma can result in Anton's syndrome.

- Anton's syndrome is associated with cortical blindness with intact anterior visual pathways.

- It commonly occurs in extensive brain injury.

\section{Plain Language Summary}

Head trauma can result in Anton's syndrome, and its exact pathophysiology is a matter debate. Anton's syndrome is associated with cortical blindness with intact anterior visual pathways, but there have been several reports suggesting that it may occur in patients with lesions to the anterior visual system, including anterior chamber, optic nerve, and optic chiasm in the background of intact visual cortex. Visual agnosia and confabulations in these cases contributed to the disconnection phenomena occurring within the consciousness awareness system located in bilateral parietal and frontal lobes and between the visual and language area. It commonly occurs in extensive brain injury.

\section{Introduction}

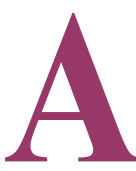

nton's syndrome is described as a denial of vision loss or visual anosognosia associated with confabulation in the setting of obvious visual loss and blindness. Head trauma, although rarely, can result in Anton's syndrome, and its exact pathophysiology is still a matter of continued debate [1]. Classically Anton's syndrome is purely associated with cortical blindness with intact anterior visual pathways, but there have been several reports suggesting that it may occur in patients with lesions to the anterior visual system, including anterior chamber, optic nerve, and optic chiasm in the background of intact visual cortex [2]. Visual agnosia and confabulations in these cases contributed to the disconnection phenomena occurring within the consciousness awareness system located in bilateral parietal and frontal lobes and between the visual and language area. It commonly occurs in extensive brain injury [3]

\section{Case Presentation}

A 26-year-old motorcycle courier with no known medical illness was admitted to the hospital following a road traffic accident whereby he had lost control of his motorcycle and skidded. He sustained a severe traumatic brain injury and concomitant injuries of multiple rib fractures complicated with bilateral pulmonary contusion and right lower extremity fractures.

Computer tomography (CT) of the head on admission showed multiple fractures involving the left frontal bone and bilateral orbital wall (Figure 1). Multiple intracranial bleedings included extensive subarachnoid hemorrhage, left frontal subdural hemorrhage, and right frontal punctuates bleeding. This picture is complicated with early obstructive hydrocephalus and cerebral edema (Figure 2).

During his inpatient stay, he was noted to have bilateral retro-orbital hematoma and ophthalmoparesis with a right afferent pupillary defect. Fundoscopy was unremarkable. However, the patient denied any visual disturbances and was not cooperative for further eye assessment as he was in Rancho Los Amigos Scale of Cognitive Functioning (RLA) level V, indicating a confusional state.

He was discharged from the hospital after three weeks stay. Upon discharge, the patient was in a wheelchair and total carer dependent, although he had good motor recovery because he was still in RLA level V. He was not allowed to bear weight following the internal fixation of his right femur fracture. The rehabilitation team trained his caregiver to provide a home-based exercise program and daily reality orientation therapy for him. In the next few days after discharge, the patient showed tremendous cognitive improvement, whereby he was in RLA level VIII. In terms of physical recovery, he was allowed for partial weight-bearing ambulation by the orthopedic team. He attended a couple of physiotherapy and occupational therapy sessions as an outpatient in which he was retrained to ambulate with walking aids and perform independent activities of daily living. However, the therapists reported that he was very clumsy and did not progress much despite a good cognitive and motor recovery. His modified Barthel index (MBI) was 70 at that time, indicating his moderate dependence. 

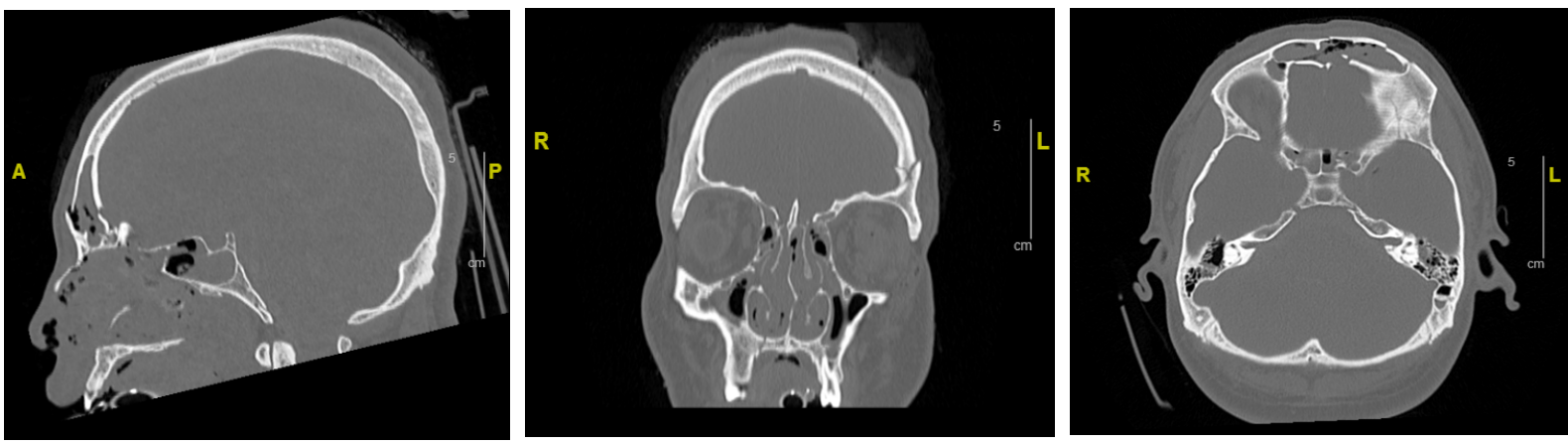

Iranian Rehabilitation \ourna

Figure 1. CT head bone window showing multiple fractures involving left frontal bone and bilateral orbital wall

The patient was followed up in our rehabilitation clinic six weeks after injury. His most striking clinical feature was severe visual acuity impairment during Mini-Mental State Examination (MMSE) performance, whereby he scored 26/30, being unable to read, write and copy. He also displayed a guessing feature when it came to naming objects. He admitted that he had mild blurring of vision, especially in the mornings but strongly maintained that he could still see objects and persons around him. Eye assessment demonstrated he had no perception of light on his right eye and 6/60 on the left. Bilateral eye movement improved with complete resolution of retroorbital hematoma. However, a right afferent pupillary defect was still present.

Further history from the caregiver showed that the patient still required partial assistance in ambulation as he frequently would walk into objects and had many episodes of near falls at home. The caregiver also revealed that the patient displayed occasional signs of visual hallucinations. He had claimed the presence of people or objects around him when in reality, there were none. In all instances, he was adamant that he was right, refusing to accept when corrected, leading to occasional displays of frustration.
At this point, the patient's rehabilitation program was redesigned with a multidisciplinary approach involving many specialties, including physical medicine rehabilitation physician, neurologist, physical therapist, occupational therapist, ophthalmologist, and psychological counselor. His therapy program consisted of orientation, mobility, and activities of daily living training with the inclusion of frequent prompts and cues to compensate for his visual deficits. These strategies were mainly to focus on functional gain. Additionally, visual restoration therapy using light stimuli was also carried out. Environmental assessment and modifications were advised accordingly to cater and create a safe living space. Simultaneously, regular psychological counseling sessions were conducted in an attempt to increase awareness and acceptance of patient's impairments to validate the necessity and importance of the conducted therapy program promoting compliance. These sessions were also helpful to address the patient's frustrations and stabilize his mood.

During the six months of post-trauma review, although the patient was noted to still have visual deficits, he had shown a better insight into his impairments. His caregiver reported that he no longer had visual hallucinations but would occasionally display an incidence of visual anosognosia. He was also more willing to accept his
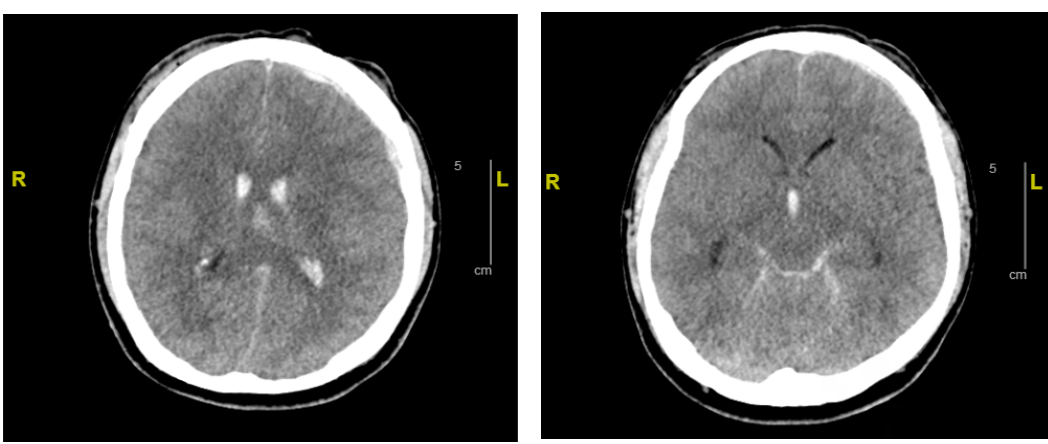

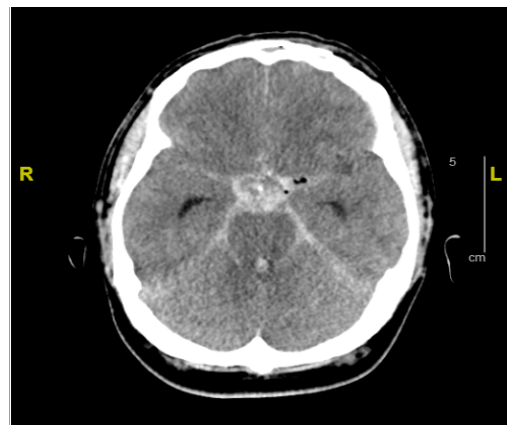

Iranian Rehabilitation Journa Figure 2. CT head brain window showing multiple intracranial bleed including extensive subarachnoid hemorrhage

Left: Frontal subdural hemorrhage; right: Frontal punctuate bleed complicated with early obstructive hydrocephalus and cerebral edema 
deficits when pointed out without showing any signs of frustration. Functionally, he had made tremendous gains whereby he had achieved an MBI score of 95, reflecting mild dependence status. The patient was now able to perform basic activities of daily living without any help and walk slowly but independently within familiar indoor settings. He still needed minimal assistance when it came to outdoor ambulation, especially in terms of navigation and avoidance of environmental obstacles owing to his visual impairment and occasional visual anosognosia. Although the patient had shown significant improvement, his family members were still instructed to provide supervision at all times that included overseeing his safety, mobility, self-care, and management of finances.

\section{Discussion}

Our patient is diagnosed with Anton's syndrome in the background of the damaged anterior visual pathway with multiple intracranial bleeds, including the frontal region and cerebral edema. This condition possibly would have led to disruption of the complex connection within his consciousness awareness system. As a result, the patient tends to fabulate answers as he cannot describe the visual stimulus owing to unawareness of the visual deficit.

In this case, the absence of the patient's complaints regarding his visual deficits, in addition to the fact that the incidence of TBI-related Anton's syndrome is uncommon, lead to its delayed diagnosis by about two months after trauma. As a result, our patient's exact impairments were missed leading to the wrong prescription of the rehabilitation program, which not only failed to yield the expected outcome but also jeopardized his safety.

Once the patient was diagnosed with Anton's syndrome, the rehabilitation strategies must be retaught meticulously. Rehabilitation of cortical blindness alone is a challenge in itself, but with anosognosia, the challenge intensifies as the patients often do not believe that they have a deficit that requires attention [4]. The patient was subjected to a mixture of compensatory and restorative strategies delivered through the combined efforts of rehabilitation medicine physicians, physiotherapists, and occupational therapists. The traditional compensatory approach included sensory rehabilitation, orientation, mobility, and environmental adaptations, which mainly helped with functional gains but not the visual function. On the other hand, visual restorative therapy (e.g., using light stimuli) is proven to improve or restore the vision through stimulation of remaining viable neurons adjacent to the damaged cerebral cortex, redirection of the visual processing via alternate pathways, or a combination of these processes based on the concept of neuroplasticity [5].

Research findings suggest that self-awareness is associated with increased participation in rehabilitation and the attainment of rehabilitation goals [6]. Therefore, regular counseling sessions by psychological counselors were incorporated alongside the patient's rehabilitation program, which may have led to the betterment of his insight on his condition, although there is a possibility of anosognosia and confabulation recovering spontaneously over time amongst the brain-injured population $[7,8]$.

It is proven that the brain is at its heightened state of plasticity during the acute phase following traumatic brain injury. As such, taking advantage of this golden period via comprehensive rehabilitation protocols undeniably propagated a patient's natural recovery by many folds. Despite showing a significant gain from baseline after rehabilitation, our young patient still has a long way to achieve the return to work and community integration. As such, the rehabilitation prescription should be tailored accordingly with smooth transitions in between to accommodate for the patient's condition and needs that evolve with time.

\section{Conclusion}

Although Anton's syndrome is a rare complication of traumatic brain injury with high risks of being overlooked during the initial neurological examination, it should be at the back of one's mind prompting necessary measures in terms of rehabilitation. Rehabilitation amongst this population is essential as it not only ensures patient's safety but also gives rise to the possibility of recovery. We have implemented a neuropsychological approach through counseling sessions in our patient and the common rehabilitation strategies applied in cortical blindness to increase the self-awareness that has appeared to benefit him. The caregiver's role is another essential aspect in the rehabilitation of patients with Anton's syndrome. They are required to provide constant supervision of patient's safety in performing daily activities at home and community level. Additionally, caregivers are also crucial in monitoring a patient's mood that could be affected by this syndrome.

\section{Ethical Considerations}

\section{Compliance with ethical guidelines}

All ethical principles are considered in this article. The participants were informed about the purpose of the research and its implementation stages. They were also as- 
sured about the confidentiality of their information and were free to leave the study whenever they wished, and if desired, the research results would be available to them.

Funding

This research did not receive any grant from funding agencies in the public, commercial, or non-profit sectors.

Conflict of interest

The author reported no conflict of interest.

\section{References}

[1] Lim SF, Chong CK, Ng P, Koh S. Transient post-traumatic cortical blindness due to bilateral occipital lobe infarcts in a multiply-injured patient: A case report. Injury Extra. 2013; 44(6):54-7. [DOI:10.1016/j.injury.2013.03.016]

[2] McDaniel KD, McDaniel LD. Anton's syndrome in a patient with posttraumatic optic neuropathy and bifrontal contusions. Archives of Neurology. 1991; 48(1):101-5. [DOI:10.1001/ archneur.1991.00530130113028] [PMID]

[3] Das JM, Naqvi IA. Anton syndrome [Internet]. 2019 [Updated 2019 April 10]. Available from: https://www.ncbi.nlm. nih.gov/books/NBK538155/

[4] Eby SA, Buchner EJ, Bryant MG, Mak HK. The rehabilitation of Anton syndrome. PM \& R: The Journal of Injury, Function, and Rehabilitation. 2012; 5(4):385-7. [DOI:10.1016/j. pmrj.2011.12.012] [PMID]

[5] Miller NR, Subramanian PS. Should visual restoration therapy be used in patients with visual field loss? Journal of Neuro-ophthalmology. 2015; 35(3):319-22. [DOI:10.1097/ WNO.0000000000000252] [PMID]

[6] Katz N, Fleming J, Keren N, Lightbody S, Hartman-Maeir A. Unawareness and/or denial of disability: Implications for occupational therapy intervention. Canadian Journal of Occupational Therapy. 2002; 69(5):281-92. [DOI:10.1177/000841 740206900504] [PMID]

[7] Fish J, Forrester J. Developing awareness of confabulation through psychological formulation: A case report and firstperson perspective. Neuropsychological Rehabilitation. 2018; 28(2):277-92. [DOI:10.1080/09602011.2017.1397031] [PMID]

[8] Acharya AB, Sánchez-Manso JC. Anosognosia [Internet]. 2021 [Updated 2021 August 1]. Available from:_https:// www.ncbi.nlm.nih.gov/books/NBK513361// 
This Page Intentionally Left Blank 